\title{
RESEARCH
}

Open Access

\section{Optical tomographic imaging of near infrared imaging agents quantifies disease severity and immunomodulation of experimental autoimmune encephalomyelitis in vivo}

Valerie L Eaton ${ }^{1 \dagger}$, Kristine O Vasquez ${ }^{2 \dagger}$, Gwendolyn E Goings ${ }^{1}$, Zoe N Hunter ${ }^{1}$, Jeffrey D Peterson ${ }^{2 *}$ and Stephen D Miller ${ }^{1 *}$

\begin{abstract}
Background: Experimental autoimmune encephalomyelitis (EAE) is an animal model that captures many of the hallmarks of human multiple sclerosis (MS), including blood-brain barrier (BBB) breakdown, inflammation, demyelination and axonal destruction. The standard clinical score measurement of disease severity and progression assesses functional changes in animal mobility; however, it does not offer information regarding the underlying pathophysiology of the disease in real time. The purpose of this study was to apply a novel optical imaging technique that offers the advantage of rapid imaging of relevant biomarkers in live animals.

Methods: Advances in non-invasive fluorescence molecular tomographic (FMT) imaging, in combination with a variety of biological imaging agents, offer a unique, sensitive and quantifiable approach to assessing disease biology in living animals. Using vascular (AngioSense 750EX) and protease-activatable cathepsin B (Cat B 680 FAST) near infrared (NIR) fluorescence imaging agents to detect BBB breakdown and inflammation, respectively, we quantified brain and spinal cord changes in mice with relapsing-remitting PLP ${ }_{139-151}$-induced EAE and in response to tolerogenic therapy.

Results: FMT imaging and analysis techniques were carefully characterized and non-invasive imaging results corroborated by both ex vivo tissue imaging and comparison to clinical score results and histopathological analysis of CNS tissue. FMT imaging showed clear differences between control and diseased mice, and immune tolerance induction by antigen-coupled PLGA nanoparticles effectively blocked both disease induction and accumulation of imaging agents in the brain and spinal cord.

Conclusions: Cat B 680 FAST and AngioSense 750EX offered the combination best able to detect disease in both the brain and spinal cord, as well as the downregulation of disease by antigen-specific tolerance. Non-invasive optical tomographic imaging thus offers a unique approach to monitoring neuroinflammatory disease and therapeutic intervention in living mice with EAE.
\end{abstract}

Keywords: Experimental autoimmune encephalomyelitis (EAE), Protease, Molecular imaging, Near infrared fluorescence

\footnotetext{
*Correspondence: jeff.peterson@perkinelmer.com; s-d-miller@northwestern.edu

${ }^{\dagger}$ Equal contributors

${ }^{2}$ Applied Biology \& In Vivo Discovery Services, PerkinElmer, Inc, 68 Elm St,

Hopkinton, Boston, MA 01748, USA

'Department of Microbiology-Immunology, Northwestern University,

Feinberg School of Medicine, 6-713 Tarry Building, 303 E Chicago Avenue,

Chicago, IL 60611, USA
}

\section{Biomed Central}

(c) 2013 Eaton et al.; licensee BioMed Central Ltd. This is an Open Access article distributed under the terms of the Creative Commons Attribution License (http://creativecommons.org/licenses/by/2.0), which permits unrestricted use, distribution, and reproduction in any medium, provided the original work is properly cited. The Creative Commons Public Domain Dedication waiver (http://creativecommons.org/publicdomain/zero/1.0/) applies to the data made available in this article, unless otherwise stated. 


\section{Background}

The experimental autoimmune encephalomyelitis (EAE) model of MS captures many of the hallmarks of the human disease $[1,2]$. EAE can be induced in specific strains of mice by immunization with CNS tissue homogenates, purified myelin proteins or peptides derived from these proteins, providing a valuable model for the assessment of the immune-related cellular and molecular contributors to disease induction or progression [3,4].

EAE is generally assessed using a subjective clinical score assessment, which does not provide information about underlying cellular or molecular processes and does not always reflect the underlying pathological changes [5]. Additional information relative to the understanding of disease can be obtained through the study of excised CNS tissues, with standard histology approaches [6], novel approaches in multiplex staining [7], confocal microscopy [8] and label-free spectroscopy [9]. A variety of clinical imaging modalities have been applied to EAE studies, including positron emission tomography (PET), singlephoton emission computed tomography (SPECT) and magnetic resonance imaging (MRI). PET and SPECT imaging, although useful in EAE [10,11], are of limited broad utility because of the expense of the imaging systems and the challenges of working with radioactive tracers. MRI imaging is also expensive and can show a poor correlation between MRI findings and clinical symptoms [12], although advances in targeted MRI contrast agents $[13,14]$ are beginning to provide more useful biological and immunological information.

Optical imaging is a relatively new modality and offers the potential advantage of rapid imaging of biological markers. Approaches with bioluminescence and fluorescence in demyelination research have relied on either transgenic luciferase-expressing mice [15] or systemically injected fluorescence imaging agents [16], respectively, relying on $2 \mathrm{D}$ surface bioluminescence or epifluorescence signals. More recent advances in fluorescence molecular tomography (FMT) offer a simple and inexpensive imaging option for preclinical research with the benefits of deep tissue detection and improved accuracy of quantification [17-19]. Here, for the first time we apply FMT in conjunction with near infrared (NIR) agents to test the hypothesis that we can detect demyelinating disease and therapeutic efficacy in the brain and spinal cord of EAE mice. A variety of NIR agents were useful in detecting changes associated with disease, including protease activatable agents to detect cathepsin and matrix metalloprotease (MMP) activity associated with inflammation (Cat B $680 \mathrm{FAST}^{\mathrm{TM}}$, ProSense ${ }^{\circ}$ 750EX and MMPSense 750 FAST), enzymatic renin activity associated with abnormal renin-angiotensin-system activity in the CNS (ReninSense $680 \mathrm{FAST}^{\mathrm{TM}}$ ) and vascular agents designed to detect blood-brain barrier breakdown (AngioSense ${ }^{\circ}$ 680, AngioSense ${ }^{\bullet}$ 750EX). Imaging and analysis techniques were carefully characterized and non-invasive imaging results corroborated by both ex vivo tissue imaging and comparison to clinical score results. Cat B 680 FAST and AngioSense 750EX offered a combination best able to detect disease in the brain and spinal cord, as well as the downregulation of disease by antigen-specific tolerance.

\section{Materials and methods}

\section{Induction and clinical evaluation of EAE}

For the PLP $139-151$-induced experimental autoimmune encephalomyelitis experiments, specific pathogen-free female SJL/J mice (6 to 8 weeks of age) were purchased from Harlan Laboratories (Indianapolis, IN) and housed at the Center for Comparative Medicine at Northwestern University (Chicago, IL) under a controlled environment (72 F; 12:12-h light-dark cycle) under specific pathogenfree conditions with water and food provided ad libitum. All experiments were performed in accordance with Northwestern University IACUC guidelines for animal care and use. Proteolipid protein (PLP) ${ }_{139-151}$ (HSLGKWLGHPDKF)

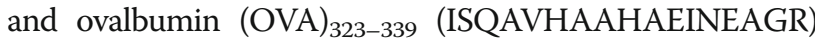
were purchased from Genemed Synthesis. EAE was elicited by immunization with $50 \mu \mathrm{g}$ of $\mathrm{PLP}_{139-151}$ in complete Freund's adjuvant (CFA) supplemented with $200 \mu \mathrm{g}$ heat-killed M. tuberculosis H37Ra (Difco Laboratories, Detroit, MI). A volume of $0.1 \mathrm{ml}$ of emulsion was distributed subcutaneously over three spots on the dorsal flanks on day 0 . Observational clinical scores for each mouse were recorded daily using a scale of $0-5$ as listed below:

Clinical Score of 0: No abnormalities

Clinical Score of 1: Limp tail or hind limb weakness

Clinical Score of 2: Both limp tail and hind limb weakness

Clinical Score of 3: Partial hind limb paralysis

Clinical Score of 4: Total hind limb paralysis

Clinical Score of 5: Moribund

\section{Tolerance induction with Ag-coupled nanoparticles}

As previously described [20], 500-nm carboxylated PLGA microparticles were purchased from Phosphorex, Inc. (Fall River, MA), and peptide antigens were attached using ECDI (1-ethyl-3-(3'-dimethylaminopropyl)carbodiimide; EMD Chemicals Inc., Gibbstown, NJ) with $0.08 \mathrm{mg}$ of peptide in the presence of $0.32 \mathrm{mg}$ ECDI per $1.0 \mathrm{mg}$ of PLG nanoparticles. Animals received intravenous injections of approximately $9 \times 10^{9}$ nanoparticles comprising $10-15 \mu$ g of peptide, depending on the peptide sequence used in the coupling reaction.

\section{Fluorescent agents for the detection of inflammation}

Six commercially available imaging agents (PerkinElmer Inc., Waltham, MA) were used to optimize EAE imaging and detect therapeutic efficacy (Table 1). AngioSense is 
Table 1 Characteristics of fluorescent imaging agents

\begin{tabular}{|c|c|c|c|c|}
\hline Agent & Description & M.W. & Peak Ex/Em & Biological activity \\
\hline Cat B 680 FAST & Cathepsin-activatable & $33 \mathrm{kDa}$ & $675 / 693$ & Macrophage/microglia \\
\hline MMPSense 750 FAST & Metalloproteinase-activatable & $43 \mathrm{kDa}$ & $749 / 775$ & Macrophage/microglia \\
\hline ProSense 750 EX & Cathepsin-activatable & $450 \mathrm{kDa}$ & $750 / 770$ & Macrophage/microglia \\
\hline ReninSense 680 FAST & Renin-activatable & $43 \mathrm{kDa}$ & $675 / 693$ & T cell RAS activity \\
\hline AngioSense 680 & Vascular agent & $250 \mathrm{kDa}$ & $680 / 700$ & BBB breakdown \\
\hline AngioSense 750 EX & Vascular agent & $70 \mathrm{kDa}$ & $750 / 770$ & BBB breakdown \\
\hline
\end{tabular}

a vascular imaging agent; ProSense and Cat B detect regions of increased lysosomal cathepsin activity (ProSense is a pan cathepsin agent, while Cat B is preferentially cleaved by cathepsin B); ReninSense FAST is activated by kidney renin.

\section{In vivo fluorescence imaging}

Mice were maintained on a low fluorescence alfalfa-free diet (Harlan 2019) recommended for fluorescence imaging, and imaging was performed at the peak of disease (d15). The mice were injected intravenously with $2 \times$ the recommended dose of fluorogenic agents (to facilitate brain biodistribution) at $\mathrm{d} 14$ and imaged $24 \mathrm{~h}$ later (Figure 1). Prior to the imaging, the animals were anesthetized with an i.p. injection of ketamine $(100 \mathrm{mg} / \mathrm{kg})$ and xylazine $(20 \mathrm{mg} / \mathrm{kg})$ in PBS, and hair from each mouse was removed using depilatory cream (Nair, Church \& Dwight Co., Ewing, NJ). The cream was applied 2-3 times, for only 4-5 min each time, to minimize harm to the skin. The animals were then imaged using the FMT $4000^{\mathrm{m}}$ fluorescence tomography in vivo imaging system (PerkinElmer, Hopkinton, MA). For whole body imaging, the anesthetized mouse was placed in a prone position on the imaging cassette to capture the spine or brain of the animal within the imaging scan field. The imaging cassette was adjusted to the proper depth to gently restrain each mouse and then inserted into the heated docking system (regulated at $\sim 37^{\circ} \mathrm{C}$ ) in the FMT imaging chamber. For imaging the head, the mouse was positioned prone on a resin block (designed to mimic optical scattering and absorption properties of the mouse's body and minimize imaging artifacts from air-containing spaces in the head, such as ear canals and sinuses) and positioned in the imaging cassette. A NIR laser diode was used to transilluminate (i.e., pass light through the body of the animal to be collected on the opposite side) each mouse's body, with signal detection occurring via a thermoelectrically cooled CCD camera placed on the opposite side of the imaged animal. Appropriate optical filters allowed collection of both fluorescence and excitation data sets, and the multiple source-detector fluorescence projections were normalized to the paired collection of laser excitation data. The entire image acquisition sequence took approximately 6-8 min per mouse. FMT 4000 epifluorescence imaging was routinely performed prior to each tomographic imaging session using built-in LED front illuminators and collection of single camera images.

Animals that were imaged in vivo were then euthanized by exsanguination following anesthesia. The mice were transcardially perfused with cold PBS, and the brains and spinal cords were removed post-mortem then imaged by epifluorescence imaging using the FMT 4000.

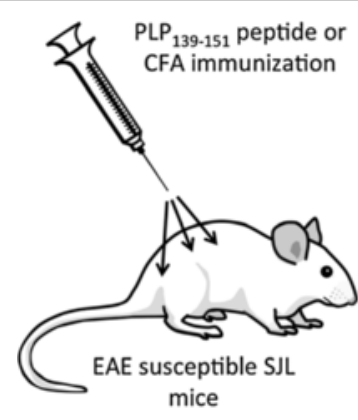

Development of CNS-directed autoimmunity IV injection of imaging agents

Enrollment Score of 2-3

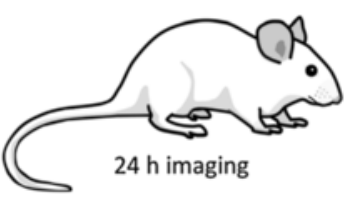

Figure 1 Experimental autoimmune encephalomyelitis induction and imaging. SJL/J mice were immunized with PLP $139-151$ in CFA as described in the Materials and Methods section. Mice were monitored for clinical signs of disease and enrolled for imaging agent injection upon achieving a score of 2 or 3 , and asymptomatic mice were excluded from imaging. Control mice (immunized with CFA only) were injected with imaging agents on day 14. All mice were imaged $24 \mathrm{~h}$ after imaging agent injection. For treatment studies, all mice (including asymptomatic mice from the positive control group) were injected on day 13 for imaging on day 14. Two separate FMT tomographic scans (head and spine) were performed, and excised brains and spinal cords were imaged by FMT epifluorescence to confirm in vivo results. 


\section{FMT reconstruction and analysis}

The collected fluorescence data sets were reconstructed by FMT 4000 system software (TruQuant ${ }^{\mathrm{TM}}$, PerkinElmer, Waltham MA) for the quantification of fluorescence signal within the head and spine. Three-dimensional regions of interest (ROI) were drawn to encompass each region, and a modest threshold was applied identically to all animals within each agent group in order to minimize background signal. Briefly, the brain ROI for each mouse was placed as a $9 \times 12 \times 6-\mathrm{mm}(\mathrm{w} \times \mathrm{h} \times \mathrm{d})$ ellipsoid in the appropriate head region, and a threshold was applied equal to $30 \%$ of the average maximum fluorescence (in $\mathrm{nM}$ ) of the control brain ROIs. For spinal cord ROIs, each was placed as a $6 \times 31 \times 4$-mm rectangular prism with thresholding applied as $10 \%$ of the average maximum fluorescence (nM) of the control spinal cord ROIs. The total amount of brain and spinal cord fluorescence (in pmoles) was automatically calculated relative to internal standards generated with known concentrations of the appropriate NIR dyes. For visualization and analysis purposes, TrueQuant software provided 3D images and tomographic slices.

\section{Statistical analysis}

Comparisons of the mean peak disease severity between any two groups of mice were analyzed by Student's $t$ test. $P$ values less than 0.05 were considered significant.

\section{Results}

Imaging CNS vascular leak in EAE with AngioSense 750EX To assess whether mouse EAE can be effectively imaged by FMT, we used an NIR vascular imaging agent, AngioSense 750EX (AS750), to detect vascular leaks in the blood-brain barrier associated with CNS disease. On day zero, SJL/J mice were immunized with PLP $139-151$ in complete Freund's adjuvant (CFA) as described in the Materials and Methods section. Injection sites (3 per mouse) were placed laterally on the backs of the mice to avoid interference with spinal cord imaging. PLP peptide-immunized mice achieved mild disease (average clinical score of 1.4) with 60\% disease incidence. Mice were injected with $4 \mathrm{nmol}$ of AS750 when disease severity in half of the mice achieved a clinical score of 2-3 (day 14), and imaging was performed $24 \mathrm{~h}$ later to allow optimal accumulation of the agent in BBB-compromised areas of the CNS.

FMT epifluorescence imaging (Figure 2A) shows that AS750 accumulated as expected within the immunization sites in both a representative CFA-immunized control and a PLP 139 -151-immunized mouse, revealing vascular leak within the resulting granulomas. Transillumination scan fields (required for FMT tomographic imaging) were positioned based upon the epifluorescence images in order to capture the head and spinal cord in two separate 2-4-min scans (Figure 2B). Tomographic fluorescence data sets were acquired, reconstructed in 3D by the FMT software and represented as 3D image overlays on the mouse image. Regions of interest (ROI) were placed to quantify brain (ellipsoidal ROI) and lumbar/sacral spinal cord (rectangular prism) fluorescent signal in three dimensions, and the resulting tomographic fluorescent images are shown in Figure 2C.

To better reveal the differences in AS750 localization within the specific anatomical regions in which disease occurs, appropriate tomographic slices were made through the ROIs of the spine (2 $\mathrm{mm}$ depth) and brain (3 $\mathrm{mm}$ depth). The resulting slice images (Figure 3A) reveal a dramatic difference between the CFA control and the EAE mouse by excluding extraneous non-CNS signal. Quantification of the entire 3D ROI data sets (i.e., all slices per ROI) from the brain and spine of all of the mice (Figure 3B) was performed with modest thresholding applied to minimize background signal in the control mice (described in Materials and Methods). Quantification revealed highly significant elevations in fluorescent signal associated with EAE. Interestingly, although disease incidence (as defined by clinical score) was $60 \%$, all of the EAE mice, including those that were asymptomatic (i.e., no hindlimb weakness or gait abnormalities), showed elevated brain fluorescence (i.e., fluorescence greater than control mean $+2 \mathrm{SD}$ ), and $60 \%$ showed elevated spine signal. These non-invasive imaging results were confirmed by ex vivo epifluorescence imaging and quantification of excised brain and spinal cord tissue (Figure 3C), with both the images and epifluorescence measurements in strong agreement with non-invasive in vivo imaging.

\section{Imaging changes in CNS protease activity in EAE mice}

Upregulated proteolytic activity is associated with a number of diseases, including cancer, inflammation, neurodegeneration, cardiovascular disease, arthritis, and diabetes [21-23]. In MS, both matrix metalloproteases (MMP) [24] and cathepsins $[25,26]$ are expressed in inflammatory cells and have been implicated as playing a role in BBB breakdown and/or direct myelin destruction. Even renin, a protease generally associated with regulation of blood pressure, has been implicated in the regulation of inflammatory cell influx [27], supporting a proposed role for the tissue-specific renin-angiotensin system in MS pathogenesis.

We next examined the potential of assessing protease activity as a biomarker of EAE severity by imaging mice at peak of acute disease with protease-activatable NIR fluorescence imaging agents: Cat B 680 FAST (CB680) for cathepsin B activity, ProSense 750EX (PS750) for pan-cathepsin activity (Cat B, L, S, K, V), MMPSense 750 FAST (MMP750) for pan-matrix metalloprotease activity (MMPs 2, 7, 9, 12, 13 and others), and ReninSense 680 FAST (RS680) for renin activity. The average clinical score of all PLP 139 -151-immunized mice for this study was 1.45 on day 14 , with disease incidence of $53 \%$. Asymptomatic 

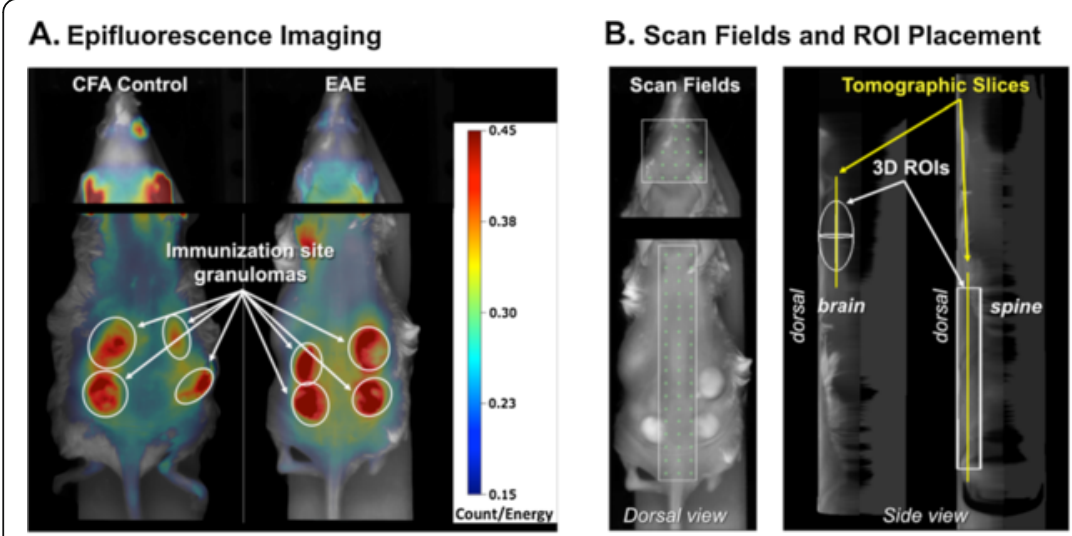

\section{Total Tomographic Fluorescence}

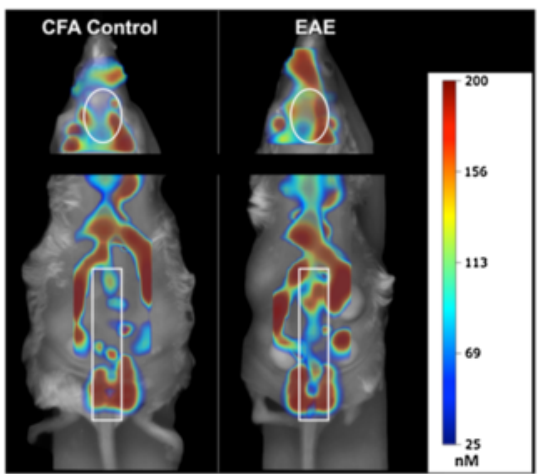

Figure 2 EAE image acquisition and analysis of AS750. (A) Whole-body epifluorescence AS750 signal was acquired to show signal within the sites of immunization. (B) FMT tomographic scan fields (left panel) show the transillumination scan pattern performed for the spine and head regions. $3 \mathrm{D} \mathrm{RO}$ l placement and sizing is shown for the separate head and spine scans (right panel). (C) Total fluorescent signal in $3 \mathrm{D}$ throughout the spine and head regions is shown to reveal the complexity of bodily signal following AS750 injection.

mice were excluded from the study, and the overtly diseased animals showed an average clinical score of 2.75 . Control mice were injected intravenously with agents 14 days after immunization with CFA; PLP $_{139-151}$-immunized mice were injected with agents on the first day that they achieved a clinical score of 2 or 3 (days 13-14). Mice were imaged and analyzed as described in the study shown in Figures 2 and 3, and the data show the fluorescence (pmol) in brain and spine and the fold change as compared to control mice (Figure 4A). In addition, isolated brain and spinal cord tissue fluorescences (counts/energy) were assessed by epifluorescence imaging (Figure 4B).

CB680 imaging showed statistically significant elevations in the brains of EAE mice (3.6-fold increase relative to controls), and this was confirmed in ex vivo tissue imaging. Interestingly, neither RS680 nor MMP750 showed significant brain elevations, despite the association of MMPs and renin in EAE and the similar molecular weights as compared to CB680. In contrast, RS680 showed statistically significant elevations in the spinal cord, confirmed by ex vivo tissue assessment. CB680 also showed spinal cord elevation as confirmed both by ex vivo imaging and histological examination showing activated cathepsin $\mathrm{B}$ expression in $\mathrm{F} 4 / 80^{+}$macrophages/microglia in a spinal cord inflammatory lesion (Figure 4C), but the variability of the in vivo imaging in this particular study prevented attainment of statistical significance. One of the most interesting findings was

\section{A. Tomographic Region Slices}

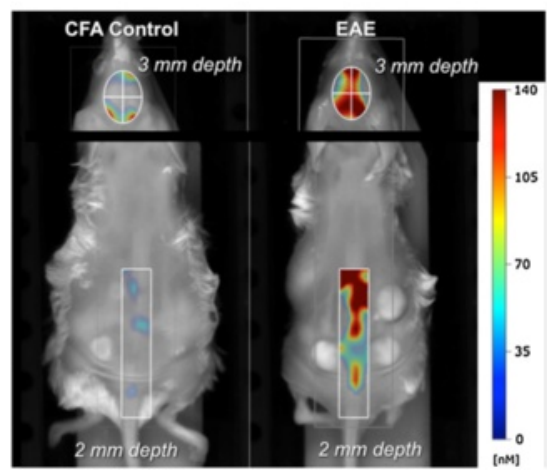

\section{B. Tomographic Quantification}

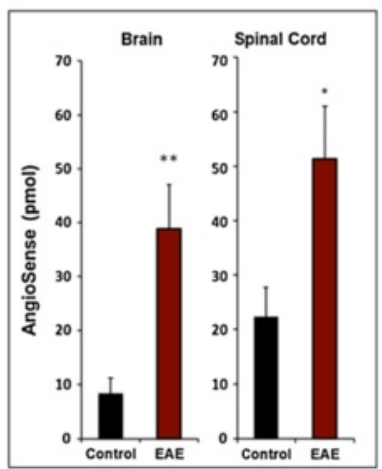

\section{Ex Vivo Tissue Imaging and Quantification}

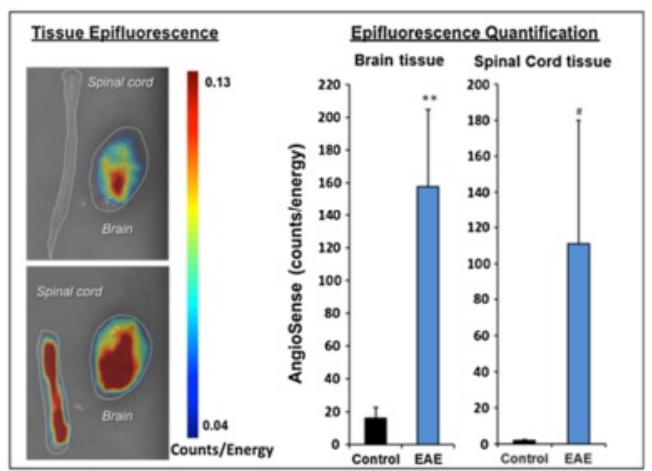

Figure 3 Quantifying BBB disruption. PLP $139-151^{-i m m u n i z e d ~ m i c e ~ w e r e ~ i n j e c t e d ~ i n t r a v e n o u s l y ~ w i t h ~ A S 750 ~ u p o n ~ a c h i e v i n g ~ a ~ c l i n i c a l ~ s c o r e ~ o f ~} 2$ or 3 and imaged by FMT 4000 using tomographic scanning capabilities. CFA control mice were injected 14 days following immunization. (A) NIR tomographic fluorescent signal in 2-3-mm slices through the spinal cord and brain regions of representative control and diseased mice. (B) NIR tomographic quantification of the fluorescent signal (mean pmol $\pm \mathrm{SE}$ ) in the brains and spinal cord of all experimental mice. (C) Brains and spinal cord tissues of the same representative mice shown in A were imaged by epifluorescence on the FMT (left panel). Epifluorescence quantification (right panel) for tissues from all mice. Study is representative of three separate experiments, $\# p<0.05,{ }^{*} p<0.01,{ }^{* *} p<0.005$ ). 


\section{A. In Vivo Imaging}
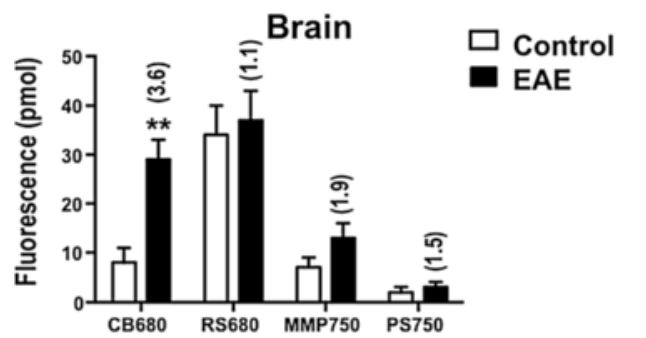

\section{B. Ex VivoTissue Imaging}

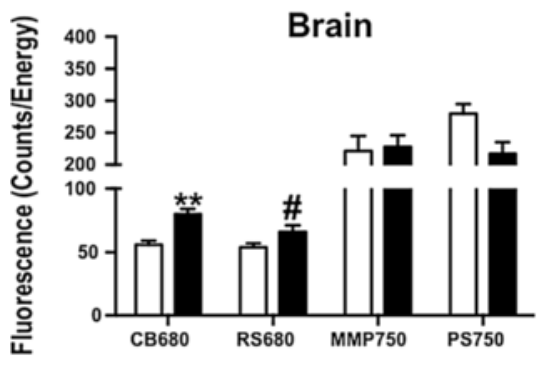

\section{Spinal Cord Histology}

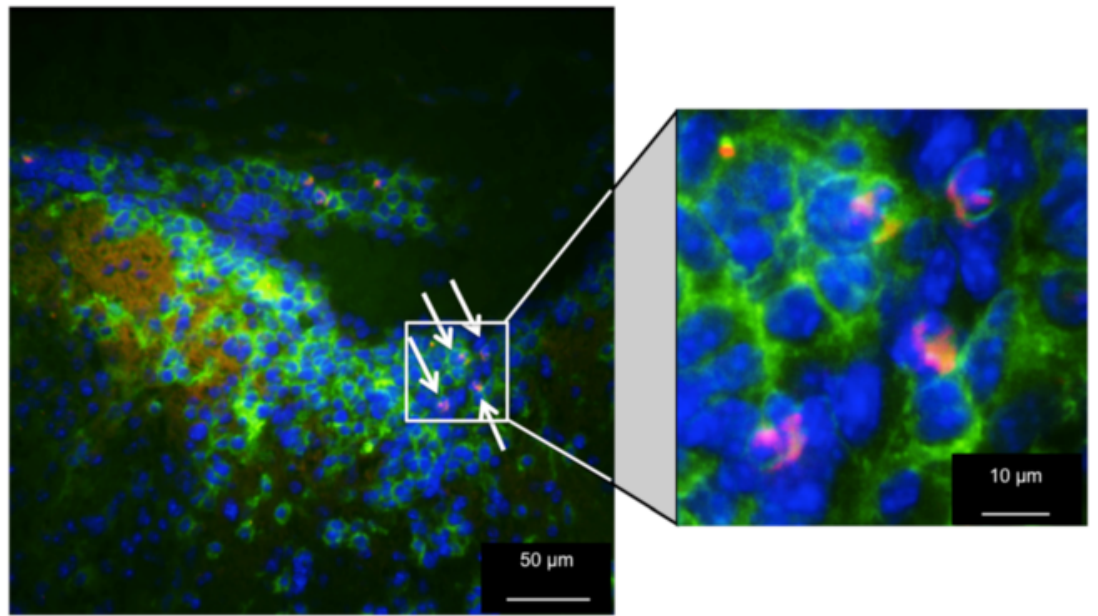

Figure 4 Imaging protease activity in vivo. $\mathrm{PLP}_{139-151}$-immunized mice were injected intravenously with various protease-activatable NIR fluorescence imaging agents [CB680 for cathepsin B activity; PS750 for pan-cathepsin activity (Cat B, L, S, K, V); MMP750 for pan-matrix metalloprotease activity (MMPs 2, 7, 9, 12, 13 and others); and RS680 for renin activity] upon achieving a clinical score of 2 or 3 and imaged by FMT 4000 using tomographic scanning capabilities. CFA control mice were injected 14 days following immunization. (A) Quantification of in vivo tomographic fluorescence data sets. (B) Quantification of epifluorescence signal from excised brains and spinal cords. Symbols indicate statistical significance ( $\left.\# p<0.05,{ }^{* *} p<0.005\right)$. Fold change compared to CFA controls is indicated in parentheses for the in vivo imaged mice. (C) Low and high power magnifications of cathepsin B expressing macrophages/microglia in a frozen section of an inflammatory lesion in the dorsal sacral spinal cord of a perfused EAE mouse injected with CB680 24 h previously. Green (anti-F4/80); red (CB680); blue (DAPI).

that the pan-cathepsin imaging agent, PS750, did not show a signal within the brain and spinal cord despite its ability to detect cathepsin $\mathrm{B}$ in a manner similar to CB680. As the molecular weight of this agent is approximately $450,000 \mathrm{Da}$, it appears that this may have affected BBB passage.
Imaging therapeutic efficacy of antigen-specific immune modulation in EAE mice

We have recently shown that intravenous injection of

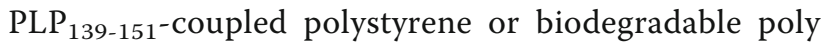
(lactic-co-glycolic acid) beads (PLP-PSB or PLP-PLGA) can decrease EAE induction and severity induced by 
PLP $_{139-151}$ immunization when using either prophylactic or therapeutic treatment regimens [20]. These particles are taken up via scavenger receptors on antigen-presenting cells to induce both pathogenic $\mathrm{T}$ cell hyporesponsiveness (anergy) and increased activity of regulatory T cells. We assessed the ability of CB680/AS750 imaging to detect tolerance-induced disease inhibition by treating mice with either OVA $_{323-339}$-PLGA (OVA tolerized) or PLP $139-151^{-}$ PLGA (PLP tolerized) 7 days prior to immunizing mice with $\mathrm{PLP}_{139-151} / \mathrm{CFA}$. The average clinical score of control OVA-tolerized mice was 2.4 by day 13 , with disease incidence over $70 \%$ (Figure 5). In contrast, both average clinical score and disease incidence were significantly decreased in PLP-tolerized mice (clinical score 0; incidence 0\%).

On day 13, all OVA- and PLP-tolerized mice were injected with a cocktail of CB680 and AS750 and imaged on day 14 (24 h later). Imaging results focused only on the signal within the spinal cord and brain regions for control OVA-tolerized (Figure 6A) and PLP-tolerized (Figure 6B) mice. Left panels show an overlay of AS750 and CB680 signal within the affected tissues in the living mice, with a clear difference apparent in the comparison of controls and tolerized mice. Very little signal is seen in the PLP-tolerized mice, whereas widespread regions of distinct and colocalized fluorescent signals are seen in the OVA-tolerized controls. As has been observed in previous studies, the brain signal in diseased mice tends to be much more variable than that in the spine regions; only half of the mice showed high brain signal. The right panels show the epifluorescence signal in the excised brain and spinal cord tissue, again represented as a two-color overlay of

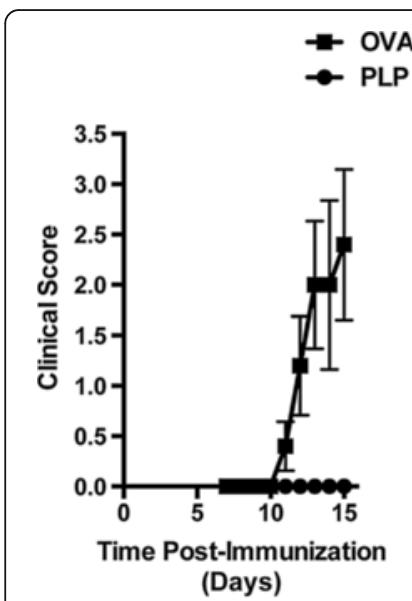

Figure 5 Clinical scores and disease incidence for control and PLP-tolerized mice. Mice were injected with $\mathrm{OVA}_{322-339-c o n j u g a t e d}$ or PLP $139-151^{-}$-conjugated PLGA nanoparticles 7 days prior to immunization with PLP $_{139-151}$ in CFA (as described in Materials and Methods). Mean clinical scores (left panel) were assessed on days 7 through 15 following immunization, and disease incidence (right panel) was determined based on mice with a score of at least 1. the two imaging agents. This ex vivo assessment correlates well with the non-invasive images acquired in the living mice.

Quantification of the two-channel fluorescent signal in EAE mice shows excellent detection of therapeutic efficacy of PLP tolerance induction when examining the spinal cord region (Figure 7) of the in vivo imaging data sets (left panels). Brain data, in contrast, showed the inherent variability of brain disease and a lack of correlation with clinical score. Thus, although there was a trend toward a decrease in brain signal with PLP-PLGA treatment, a much larger study would be required to assess this. The ex vivo brain and spinal cord tissue imaging by epifluorescence (right panels) confirmed in vivo observations and showed marginal statistical significance for AS750 brain signal.

\section{Discussion}

Rodent models of EAE are used in research and drug development because of their similar pathology and/or pathogenesis to human MS and their ease of use and reproducibility. These models share a number of important morphologic and immunologic features with human MS, and they have contributed greatly to the overall knowledge of immune-mediated myelin destruction. Damage occurs in three phases; first some abnormal areas of tissue damage appear in the brain and spinal cord, followed later by leaks in the blood-brain barrier and then immune cell infiltration ultimately leading to demyelination [28]. EAE disease assessment relies heavily upon subjective clinical scoring of symptoms that capture little information regarding the molecular processes that drive these phases of disease. More detailed mechanistic information is typically obtained by histologic analyses of affected brain and spinal cord tissues.

New vascular and protease-activatable molecular imaging agents, as well as current improvements in imaging methods, provide an opportunity to gather non-invasive in vivo biological data regarding disease severity, progression and response to therapy. BBB compromise in EAE is well characterized [29], and AngioSense, a validated NIR vascular imaging agent [30,31], offers an opportunity for in vivo imaging of vascular leak into the CNS. In addition, as proteases play a central role in cancer and inflammatory disease processes, we anticipated that protease-activatable NIR imaging agents [32,33] could serve as a sensitive means for detecting neuroinflammation in EAE and changes with therapeutic intervention. Protease-activatable agents have been used to image protease increases in a number of disease states, including cancer [34,35], asthma $[36,37]$, atherosclerosis $[38,39]$ and arthritis $[40,41]$. However, it remained to be determined whether this collection of NIR imaging agents can pass the BBB and effectively detect disease within the CNS. 


\section{A. OVA-tolerized Controls}
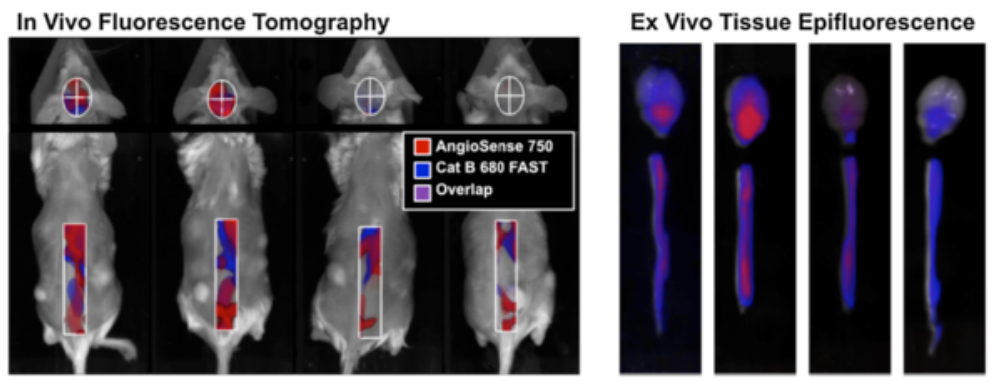

\section{B. PLP-tolerized}

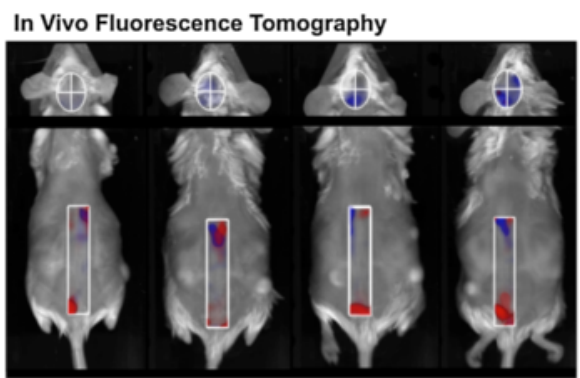

Ex Vivo Tissue Epifluorescence

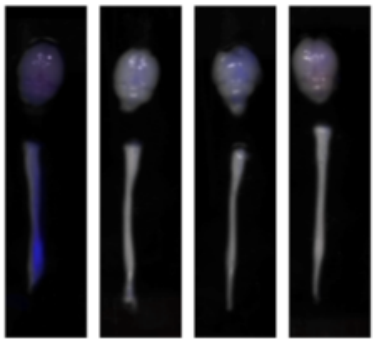

Figure 6 NIR tomographic multiplex imaging AS750 and CB680 in control and PLP-tolerized mice. (A) Tomographic images of vascular leak and cathepsin B activity in brain and lumbar/sacral spinal cord of control OVA-tolerized mice (left panel). Extraneous non-CNS signal was excluded for clarity. Right panel shows CB680/AS750 signal from excised brain and spinal cord tissue. (B) Tomographic images of vascular leak and cathepsin B activity in brain and lumbar/sacral spinal cord of control PLP-tolerized mice (left panel). Extraneous non-CNS signal was excluded for clarity. Right panel shows CB680/AS750 signal from excised brain and spinal cord tissue. Tomographic images from $\mathbf{A}$ and $\mathbf{B}$ are represented as fluorescence isosurfaces showing signal $\geq 65 \mathrm{nM}$ (CB680 brain), $\geq 200 \mathrm{nM}$ (AS7750 brain) and $\geq 100 \mathrm{nM}$ (CB680/AS750 spine). Epifluorescence images are represented with the same monochrome color scales for both control and tolerized mouse tissues.

For the first time, we show the benefit of fluorescence molecular tomographic imaging in a mouse model of MS and demonstrate that tomography can not only provide images reflecting the disease severity, but also provide multiplex quantitative measurements (Figures 2, 3, 5 and 6). The first challenge in establishing a method for non-invasive EAE imaging of the brain and spinal cord was to determine whether our multiple potential agents, ranging in size from $33-450 \mathrm{kDa}$, could penetrate the $\mathrm{BBB}$ of mice. A number of preliminary studies with larger molecular
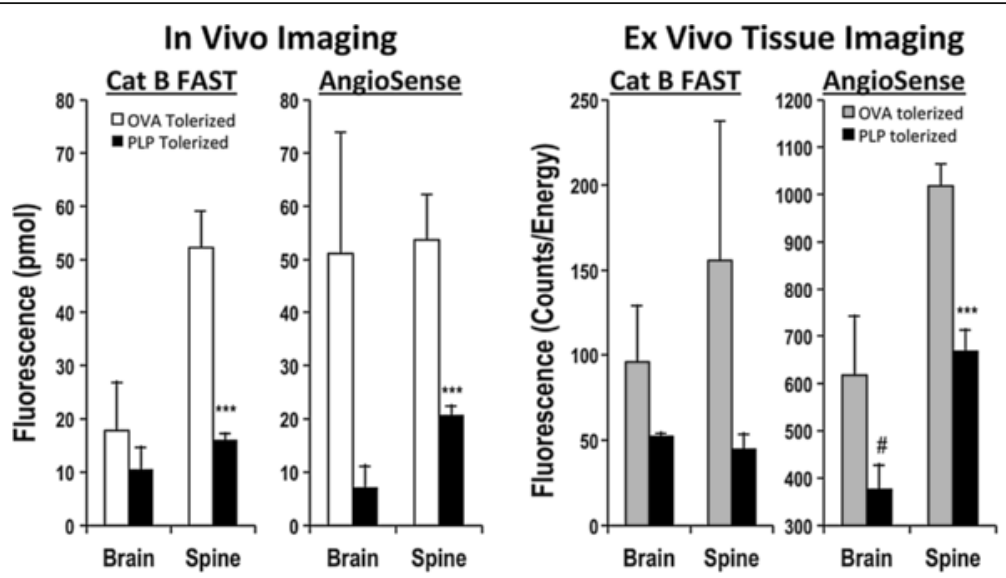

Figure 7 Quantifying PLP-specific tolerance induction in EAE. Fluorescence tomography and tissue epifluorescence were quantified for control and PLP-tolerized mice from the study represented in Figures 4 and 5. The left panel shows the quantification of in vivo tomographic fluorescence data sets. The right panel shows the quantification of epifluorescence signal from excised brains and spinal cords. Symbols indicate statistical significance $\left(\# p<0.05,{ }^{* *} p<0.0001\right)$. 
weight AS750 (250 kDa) and activatable PS680 (450 kDa) revealed superior CNS accumulation by the somewhat smaller AS750 (data not shown), consistent with our findings in these studies. This led us to perform much of our early method validation with AS750, defining ROI shapes and sizes as well as optimal fluorescence thresholding approaches that yielded the technique described in Materials and Methods. All of our data to date have revealed considerable variability in brain disease (as also shown in the studies in Figures 4 and 6) and have further suggested that brain signal decreases dramatically in severely affected animals (clinical score of 4). Interestingly, PLP peptide-immunized animals with clinical scores of 0 or 1 often show as much brain fluorescence as mice with clinical scores of 2 or 3 . This suggests that there can be some form of active disease occurring even in mice with no clinical symptoms, a finding that is not surprising as EAE clinical scores are mostly an assessment of spinal cord damage. Spine signal, on the other hand, generally correlates reasonably well with the clinical score but with more variability in mice with clinical scores of 4 . Despite careful care of these animals, some of this decline in seriously diseased animals may be due to overall physiologic changes caused by dehydration and decreased circulation in these animals.

Optical tomographic imaging using NIR imaging agents in EAE has the potential for use in drug discovery research by virtue of deep tissue penetration, quantitative readout (pmol rather than light intensity) and the pairing with NIR imaging agents that detect the cellular participants in the underlying disease pathology. For the past 25 years, the Miller laboratory has explored specific T cell tolerance, induced by antigen-coupled splenocytes, as a means to both treat disease [42-45] and probe mechanisms of immunopathogenesis [46,47]. Recent advances in techniques for inducing $\mathrm{T}$ cell tolerance to specific neuroantigen epitopes have yielded an approach using antigen-coupled biodegradable PLGA nanoparticles to probe mechanisms of immune tolerance induction [20]. We applied our FMT imaging techniques with two of the best NIR agents identified from our validation studies, and were able to detect and quantify decreases in spinal cord vascular leak and cathepsin B activity in agreement with the effects on clinical scores (Figures 4, 5, and 6). Brain signal was variable, and half of the OVA-tolerized control mice showed little brain fluorescent signal with either agent. This highlights the variability of brain disease, but it also shows that vascular leak changes accompany cathepsin B changes as would be expected given that BBB compromise generally accompanies neuroinflammation. We were also able to confirm abnormal renin activity in EAE, in agreement with reports that a CNS renin-angiotensin system may be involved in the regulation of the disease [27]. Unfortunately, a similar agent for the detection of matrix metalloprotease activity
(MMP750) was unable to detect changes in EAE despite the presence of MMP-secreting inflammatory cells. It is unclear whether unknown physical/chemical properties of this agent limited its CNS penetration or activity in vivo. Future studies will assess these agents, and additional NIR imaging agents, for their utility to detect disease as well as their sensitivity in detecting very early, subclinical disease.

Our studies have built upon advances in imaging technology to establish non-invasive optical tomographic imaging as a robust and accurate means of assessing BBB compromise and neuroinflammation in EAE in vivo. This technology offers a powerful tool for both basic neuroinflammation research and drug discovery and development by enabling the non-invasive monitoring of cellular processes that also drive human MS pathology. FMT thus should provide a rapid means for assessing and characterizing therapeutics for clinical evaluation.

\section{Abbreviations}

BBB: Blood-brain barrier; CAT: Cathepsin; CFA: Complete Freund's adjuvant; EAE: Experimental autoimmune encephalomyelitis; ECDI: 1-Ethyl-3-(3'-dimethylaminopropyl)carbodiimide; FMT: Fluorescence molecular tomographic imaging; MMP: Matrix metalloprotease; MRI: Magnetic resonance imaging; MS: Multiple sclerosis; NIR: Near infrared; OVA: Ovalbumin; PET: Positron emission tomography; PLGA: Poly (lactide-co-glycolic) acid; PLP: Proteolipid protein; ROI: Regions of interest; SPECT: Single photon emission computed tomography.

\section{Competing interests}

Peterson and Vasquez are funded by PerkinElmer Inc., and this is clearly stated in the manuscript. The research documents the utility of PerkinElmer imaging agents and imaging technology in addressing specific biological questions in experimental autoimmune encephalomyelitis. The expectation is to further increase scientific interest in optical imaging in general and in PerkinElmer imaging agents and technology. As the technology (and imaging agents) have been around for several years (and have been published to some extent), the company expects no major financial gain as a result of publication beyond a modest increase in awareness in proportion to the robustness of the research. Northwestern University, Feinberg School of Medicine, employees have no financial stake in PerkinElmer Inc. The authors hold no patents relevant to the manuscript, and no patent applications are being submitted (or planned to be submitted). No reimbursement or funding relating to potential patents has been received. PerkinElmer currently holds patents for the technology used in the research.

\section{Authors' contributions}

JDP and SDM designed the research, analyzed data and wrote the paper; VLE, KOV, GEG and ZNH performed the experiments. All authors read and approved the final manuscript.

\section{Acknowledgements}

Supported in part by internal funding from PerkinElmer, Inc., and a grant from the Myelin Repair Foundation.

Received: 25 September 2013 Accepted: 7 November 2013

Published: 15 November 2013

\section{References}

1. Constantinescu CS, Farooqi N, O'Brien K, Gran B: Experimental autoimmune encephalomyelitis (EAE) as a model for multiple sclerosis (MS). Br J Pharmacol 2011, 164:1079-1106.

2. Gold R, Linington C, Lassmann H: Understanding pathogenesis and therapy of multiple sclerosis via animal models: 70 years of merits and culprits in experimental autoimmune encephalomyelitis research. Brain 2006, 129:1953-1971. 
3. Steinman L: Multiple sclerosis: a two-stage disease. Nat Immunol 2001, 2:762-764.

4. Rangachari M, Kuchroo VK: Using EAE to better understand principles of immune function and autoimmune pathology. J Autoimmun 2013 http://dx.doi.org/10.1016/j.jaut.2013.1006.1008.

5. Kallen B, Nilsson O: Dissociation between histological and clinical signs of experimental auto-immune encephalomyelitis. Acta Pathol, Microbiol, et Immunol Scand - Sec A, Pathol 1986, 94:159-164.

6. Kuerten S, Addicks K, Lehmann PV: Studies on the CNS histopathology of EAE and its correlation with clinical and immunological parameters. In Experimental Autoimmune Encephalomyelitis - Models, Disease Biology and Experimental Therapy. Edited by Weissert R. InTech; 2012:21-46.

7. Eckhardt J, Ostalecki C, Kuczera K, Schuler G, Pommer AJ, Lechmann M: Murine whole-organ immune cell populations revealed by multi-epitope-ligand cartography. J Histochem Cytochem 2013, 61:125-133.

8. Paul D, Cowan AE, Ge S, Pachter JS: Novel 3D analysis of Claudin-5 reveals significant endothelial heterogeneity among CNS microvessels. Microvasc Res 2013, 86:1-10.

9. Fu Y, Frederick TJ, Huff TB, Goings GE, Miller SD, Cheng JX: Paranodal myelin retraction in relapsing experimental autoimmune encephalomyelitis visualized by coherent anti-Stokes Raman scattering microscopy. J Biomed Opt 2011, 16:106006.

10. Radu CG, Shu CJ, Shelly SM, Phelps ME, Witte ON: Positron emission tomography with computed tomography imaging of neuroinflammation in experimental autoimmune encephalomyelitis. Proc Natl Acad Sci USA 2007, 104:1937-1942.

11. Abourbeh G, Theze B, Maroy R, Dubois A, Brulon V, Fontyn $Y$, Dolle F, Tavitian B, Boisgard R: Imaging microglial/macrophage activation in spinal cords of experimental autoimmune encephalomyelitis rats by positron emission tomography using the mitochondrial $18 \mathrm{kDa}$ translocator protein radioligand [(1)(8)F]DPA-714. J Neurosci 2012, 32:5728-5736.

12. Barkhof F: MRI in multiple sclerosis: correlation with expanded disability status scale (EDSS). Mult Scler 1999, 5:283-286.

13. Chen JW, Breckwoldt MO, Aikawa E, Chiang G, Weissleder R: Myeloperoxidase-targeted imaging of active inflammatory lesions in murine experimental autoimmune encephalomyelitis. Brain 2008 131:1123-1133.

14. Serres S, Mardiguian S, Campbell SJ, McAteer MA, Akhtar A, Krapitchev A, Choudhury RP, Anthony DC, Sibson NR: VCAM-1-targeted magnetic resonance imaging reveals subclinical disease in a mouse model of multiple sclerosis. FASEB J 2011, 25:4415-4422.

15. Luo J, Ho P, Steinman L, Wyss-Coray T: Bioluminescence in vivo imaging of autoimmune encephalomyelitis predicts disease. J Neuroinflammation 2008, 5:6.

16. Wang C, Wu C, Popescu DC, Zhu J, Macklin WB, Miller RH, Wang Y: Longitudinal near-infrared imaging of myelination. J Neurosci 2011, 31:2382-2390.

17. Ntziachristos $V$, Weissleder R: Experimental three-dimensional fluorescence reconstruction of diffuse media by use of a normalized Born approximation. Opt Lett 2001, 26:893-895.

18. Schulz RB, Ripoll J, Ntziachristos V: Experimental fluorescence tomography of tissues with noncontact measurements. IEEE Trans Med Imaging 2004, 23:492-500

19. Mohajerani P, Adibi A, Kempner J, Yared W: Compensation of optical heterogeneity-induced artifacts in fluorescence molecular tomography: theory and in vivo validation. J Biomed Opt 2009, 14:034021.

20. Getts DR, Martin AJ, McCarthy DP, Terry RL, Hunter ZN, Yap WT, Getts MT, Pleiss M, Luo X, King NJ, Shea LD, Miller SD: Microparticles bearing encephalitogenic peptides induce T-cell tolerance and ameliorate experimental autoimmune encephalomyelitis. Nat Biotechnol 2012, 30:1217-1224.

21. Turk B: Targeting proteases: successes, failures and future prospects. Nat Rev Drug Discov 2006, 5:785-799.

22. Vasiljeva O, Reinheckel T, Peters C, Turk D, Turk V, Turk B: Emerging roles of cysteine cathepsins in disease and their potential as drug targets. Curr Pharm Des 2007, 13:387-403.

23. Kobori $H$, Nangaku M, Navar LG, Nishiyama A: The intrarenal renin-angiotensin system: from physiology to the pathobiology of hypertension and kidney disease. Pharmacol Rev 2007, 59:251-287.

24. Rosenberg GA: Matrix metalloproteinases in neuroinflammation. Glia 2002, 39:279-291.

25. Bever CT Jr, Garver DW: Increased cathepsin B activity in multiple sclerosis brain. J Neurol Sci 1995, 131:71-73.
26. Haves-Zburof D, Paperna T, Gour-Lavie A, Mandel I, Glass-Marmor L, Miller A: Cathepsins and their endogenous inhibitors cystatins: expression and modulation in multiple sclerosis. J Cell Mol Med 2011, 15:2421-2429.

27. Stegbauer J, Lee DH, Seubert S, Ellrichmann G, Manzel A, Kvakan H, Muller DN, Gaupp S, Rump LC, Gold R, Linker RA: Role of the renin-angiotensin system in autoimmune inflammation of the central nervous system. Proc Natl Acad Sci USA 2009, 106:14942-14947.

28. Goodkin DE, Rooney WD, Sloan R, Bacchetti P, Gee L, Vermathen M, Waubant E, Abundo M, Majumdar S, Nelson S, Weiner MW: A serial study of new MS lesions and the white matter from which they arise Neurology 1998, 51:1689-1697.

29. Bennett J, Basivireddy J, Kollar A, Biron KE, Reickmann P, Jefferies WA, McQuaid S: Blood-brain barrier disruption and enhanced vascular permeability in the multiple sclerosis model EAE. J Neuroimmuno/ 2010, 229:180-191.

30. Zhang Q, Bindokas V, Shen J, Fan H, Hoffman RM, Xing HR: Time-course imaging of therapeutic functional tumor vascular normalization by antiangiogenic agents. Mol Cancer Ther 2011, 10:1173-1184.

31. Stelter L, Evans MJ, Jungbluth AA, Longo VA, Zanzonico P, Ritter G, Bomalaski JS, Old L, Larson SM: Imaging of tumor vascularization using fluorescence molecular tomography to monitor arginine deiminase treatment in melanoma. Mol Imaging 2013, 12:67-73.

32. Mahmood U, Tung CH, Bogdanov A Jr, Weissleder R: Near-infrared optical imaging of protease activity for tumor detection. Radiology 1999, 213:866-870.

33. Weissleder R, Tung $\mathrm{CH}$, Mahmood U, Bogdanov A Jr: In vivo imaging of tumors with protease-activated near-infrared fluorescent probes. Nat Biotechnol 1999, 17:375-378.

34. Bremer C, Bredow S, Mahmood U, Weissleder R, Tung CH: Optical imaging of matrix metalloproteinase-2 activity in tumors: feasibility study in a mouse model. Radiology 2001, 221:523-529.

35. Kirsch DG, Dinulescu DM, Miller JB, Grimm J, Santiago PM, Young NP, Nielsen GP, Quade BJ, Chaber CJ, Schultz CP, Takeuchi O, Bronson RT, Crowley D, Korsmeyer SJ, Yoon SS, Hornicek FJ, Weissleder R, Jacks T: A spatially and temporally restricted mouse model of soft tissue sarcoma. Nat Med 2007, 13:992-997.

36. Korideck H, Peterson JD: Noninvasive quantitative tomography of the therapeutic response to dexamethasone in ovalbumin-induced murine asthma. J Pharmacol Exp Ther 2009, 329:882-889.

37. Cortez-Retamozo V, Swirski FK, Waterman P, Yuan H, Figueiredo JL, Newton AP, Upadhyay R, Vinegoni C, Kohler R, Blois J, Smith A, Nahrendorf M, Josephson L, Weissleder R, Pittet MJ: Real-time assessment of inflammation and treatment response in a mouse model of allergic airway inflammation. J Clin Invest 2008, 118:4058-4066.

38. Deguchi JO, Aikawa M, Tung CH, Aikawa E, Kim DE, Ntziachristos V, Weissleder R, Libby P: Inflammation in atherosclerosis: visualizing matrix metalloproteinase action in macrophages in vivo. Circulation 2006, 114:55-62.

39. Nahrendorf M, Waterman P, Thurber G, Groves K, Rajopadhye M, Panizzi P Marinelli B, Aikawa E, Pittet MJ, Swirski FK, Weissleder R: Hybrid in vivo FMT-CT imaging of protease activity in atherosclerosis with customized nanosensors. Arterioscler Thromb Vasc Biol 2009, 29:1444-1451.

40. Izmailova ES, Paz N, Alencar $\mathrm{H}$, Chun M, Schopf L, Hepperle M, Lane JH, Harriman G, Xu Y, Ocain T, Weissleder R, Mahmood U, Healy AM, Jaffee B: Use of molecular imaging to quantify response to IKK-2 inhibitor treatment in murine arthritis. Arthritis Rheum 2007, 56:117-128.

41. Peterson JD, Labranche TP, Vasquez KO, Kossodo S, Melton M, Rader R, Listello JT, Abrams MA, Misko TP: Optical tomographic imaging discriminates between disease-modifying anti-rheumatic drug (DMARD) and non-DMARD efficacy in collagen antibody-induced arthritis. Arthritis Res Ther 2010, 12:R105.

42. Kennedy MK, Tan LJ, Miller SD, Dal Canto MC: Regulation of the effector stages of experimental autoimmune encephalomyelitis via neuroantigen-specific tolerance induction. J Immunol 1990, 145:117-126.

43. Tan $\sqcup$, Kennedy MK, Dal Canto MC, Miller SD: Successful treatment of paralytic relapses in adoptive experimental autoimmune encephalomyelitis via neuroantigen-specific tolerance. J Immunol 1991, 147:1797-1802.

44. Tan $\sqcup$, Kennedy MK, Miller SD: Regulation of the effector stages of experimental autoimmune encephalomyelitis via neuroantigen-specific tolerance induction. II. Fine specificity of effector T cell inhibition. J Immunol 1992, 148:2748-2755.

45. Getts DR, Turley DM, Smith CE, Harp CT, McCarthy D, Feeney EM, Getts MT, Martin AJ, Luo X, Terry RL, King NJ, Miller SD: Tolerance induced by apoptotic antigen-coupled leukocytes is induced by PD-L1+ and IL-10-producing 
splenic macrophages and maintained by T regulatory cells. J Immunol 2011, 187:2405-2417.

46. McRae BL, Vanderlugt CL, Dal Canto MC, Miller SD: Functional evidence for epitope spreading in the relapsing pathology of experimental autoimmune encephalomyelitis. J Exp Med 1995, 182:75-85.

47. Vanderlugt CL, Eagar TN, Neville KL, Nikcevich KM, Bluestone JA, Miller SD: Pathologic role and temporal appearance of newly emerging autoepitopes in relapsing experimental autoimmune encephalomyelitis. J Immunol 2000, 164:670-678.

doi:10.1186/1742-2094-10-138

Cite this article as: Eaton et al.: Optical tomographic imaging of near infrared imaging agents quantifies disease severity and

immunomodulation of experimental autoimmune encephalomyelitis

in vivo. Journal of Neuroinflammation 2013 10:138.

\section{Submit your next manuscript to BioMed Central and take full advantage of:}

- Convenient online submission

- Thorough peer review

- No space constraints or color figure charges

- Immediate publication on acceptance

- Inclusion in PubMed, CAS, Scopus and Google Scholar

- Research which is freely available for redistribution 